\title{
New Heavy Exotic Hadrons
}

\author{
Chengping Shen ${ }^{* \dagger}$ \\ Graduate School of Science, Nagoya University, Nagoya, Japan \\ E-mail: Shencpephys.hawaii.edu
}

\begin{abstract}
We review recent studies on exotic states at the Belle experiment. The results include: (1) The measurement of the cross sections of $\gamma \gamma \rightarrow \omega \phi, \phi \phi$, and $\omega \omega$ for masses that range from threshold to $4.0 \mathrm{GeV}$. In addition to signals from well established spin-zero and spin-two charmonium states, there are clear resonant structures below charmonium threshold, which have not been previously observed. We report a spin-parity analysis for the new structures; (2) No $X(3872)$ signal is observed in $\eta J / \psi$ or $\gamma \chi_{c 1}$ mode in $B$ decays. A narrow peak at $3823.5 \mathrm{MeV} / \mathrm{c}^{2}$ (named as $\left.\psi_{2}\right)$ to $\gamma \chi_{c 1}$ with a significance of 4.2 standard deviations including systematic uncertainty is observed in $B^{ \pm} \rightarrow K^{ \pm} \gamma \chi_{c 1}$; (3) The bottomonium states $h_{b}(1 P), h_{b}(2 P)$ and $\Upsilon(1 D)$ are observed in the reaction $e^{+} e^{-} \rightarrow \pi^{+} \pi^{-}+X$; (4) The observation of two narrow charged structures (named as $Z_{b}(10610)$ and $\left.Z_{b}(10650)\right)$ in the mass spectra of the $\pi^{ \pm} \Upsilon(n S)(n=1,2,3)$ and $\pi^{ \pm} h_{b}(m P)$ $(m=1,2)$ pairs that are produced in association with a single charged pion in $\mathrm{Y}(5 \mathrm{~S})$ decays.
\end{abstract}

Sixth International Conference on Quarks and Nuclear Physics,

April 16-20, 2012

Ecole Polytechnique, Palaiseau, Paris

*Speaker.

$\dagger$ On behalf of the Belle collaboration and supported by a Grant-in-Aid for Scientific Research on Innovative Areas "Elucidation of New Hadrons with a Variety of Flavors" from the ministry of Education, Culture, Sports, Science and Technology of Japan and a Grant-in-Aid for for Young Scientists (B) under contract 24740158. 


\section{Introduction}

In hadronic physics, the best understood quark-antiquark systems are heavy quarkonia, i.e, $c \bar{c}$ or $b \bar{b}$ mesons. The discovery of the missing $c \bar{c}$ or $b \bar{b}$ states and the precise measurements of properties of the already observed ones are important.

The QCD-motivated models predict the existence of hadrons of more complex structure than conventional mesons or baryons, such as hybrids, multiquark states of either molecular, tetraquark or hadrocharmonium configuration. As the conventional hadron spectrum is much cleaner than the dense spectrum of light states, exotic states containing $c \bar{c}$ or $b \bar{b}$ are expected to be identified more easily than the ones predicted in the light spectrum. Any resonance observed in addition to predicted multiplets might give a hint of such an exotic spectroscopy.

Some of the recently observed charmonium-like or bottomonium-like XYZ states could be candidates for the exotic hadrons mentioned. However most of them still await confirmation or their properties need to be further studied before any decisive interpretation is made. Here, we review some recent results on the exotic hadrons from Belle experiment.

\section{Observation of new resonant structures in $\gamma \gamma \rightarrow \omega \phi, \phi \phi$ and $\omega \omega$}

Recently a clear signal for a new state $X(3915) \rightarrow \omega J / \psi$ [四] and evidence for another state $X(4350) \rightarrow \phi J / \psi[[]$ ] have been reported, thereby introducing new puzzles to charmonium or charmonium-like spectroscopy. It is natural to extend the above theoretical picture to similar states coupling to $\omega \phi, \omega \omega$ or $\phi \phi$.

The measurements of the cross sections for $\gamma \gamma \rightarrow V V$ [B] , where $V V=\omega \phi, \phi \phi$ and $\omega \omega$, are based on an analysis of an $870 \mathrm{fb}^{-1}$ data sample taken at or near the $\Upsilon(n S)(n=1, \ldots, 5)$ resonances with the Belle detector operating at the KEKB asymmetric-energy $e^{+} e^{-}$collider.

After event selections, clear $\omega$ or $\phi$ signal is observed. The magnitude of the vector sum of the final particles' transverse momenta in the $e^{+} e^{-}$center-of-mass (C.M.) frame, $\left|\sum \vec{P}_{t}^{*}\right|$, which approximates the transverse momentum of the two-photon-collision system, is used as a discriminating variable to separate signal from background. We obtain the number of $V V$ events in each $V V$ invariant mass bin by fitting the $\left|\sum \vec{P}_{t}^{*}\right|$ distribution between zero and $0.9 \mathrm{GeV} / c$. The resulting

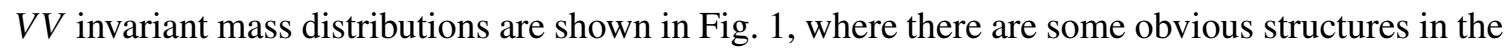
low $V V$ invariant mass region.
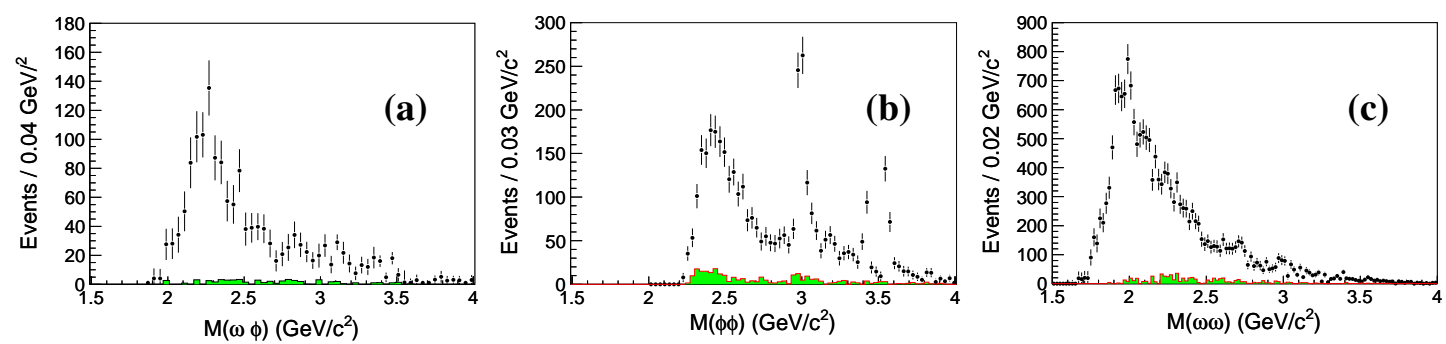

Figure 1: The (a) $\omega \phi$, (b) $\phi \phi$ and (c) $\omega \omega$ invariant mass distributions. The shaded histograms are from the corresponding normalized sidebands, which will be subtracted in calculating the final cross sections.

Two-dimensional (2D) angular distributions are investigated to obtain the $J^{P}$ of the structures. In the process $\gamma \gamma \rightarrow V V$, five angles are kinematically independent. We choose $z, z^{*}, z^{* *}, \phi^{*}$, and 
$\phi^{* *}$ [四] and use the transversity angle $\left(\phi_{T}\right)$ and polar-angle product $\left(\Pi_{\theta}\right)$ variables to analyze the angular distributions. They are defined as $\phi_{T}=\left|\phi^{*}+\phi^{* *}\right| / 2 \pi, \Pi_{\theta}=\left[1-\left(z^{*}\right)^{2}\right]\left[1-\left(z^{* *}\right)^{2}\right]$.

We obtain the number of signal events by fitting the $\left|\sum \vec{P}_{t}^{*}\right|$ distribution in each $\phi_{T}$ and $\Pi_{\theta}$ bin in the $2 \mathrm{D}$ space, which is divided into $4 \times 4,5 \times 5$, and $10 \times 10$ bins for $\omega \phi, \phi \phi$, and $\omega \omega$, respectively, for $M(V V)<2.8 \mathrm{GeV} / c^{2}$, in some wider $V V$ mass bins as shown in Fig. $\square$. The obtained 2D angular distribution data are fitted with the signal shapes from MC-simulated samples with different $J^{P}$ assumptions $\left(0^{+}, 0^{-}, 2^{+}, 2^{-}\right)$. We find: (1) for $\omega \phi: 0^{+}\left(S\right.$-wave) or $2^{+}(S$-wave) can describe data with $\chi^{2} / n d f=1.1$ or 1.2 , while a mixture of $0^{+}\left(S\right.$-wave) and $2^{+}$( $S$-wave) describes data with $\chi^{2} / n d f=0.9$ ( $n d f$ is the number of degrees of freedom); (2) for $\phi \phi$ : a mixture of $0^{+}\left(S\right.$-wave) and $2^{-}\left(P\right.$-wave) describes data with $\chi^{2} / n d f=1.3$; and (3) for $\omega \omega$ : a mixture of $0^{+}(S$-wave $)$ and $2^{+}(S$-wave $)$ describes data with $\chi^{2} / n d f=1.3$.

The $\gamma \gamma \rightarrow V V$ cross sections are shown in Fig. $\square$. The cross sections for different $J^{P}$ values as a function of $M(V V)$ are also shown in Fig. ㅁ. While there are substantial spin-zero components in all three modes, there are also significant spin-two components, at least in the $\phi \phi$ and $\omega \omega$ modes.
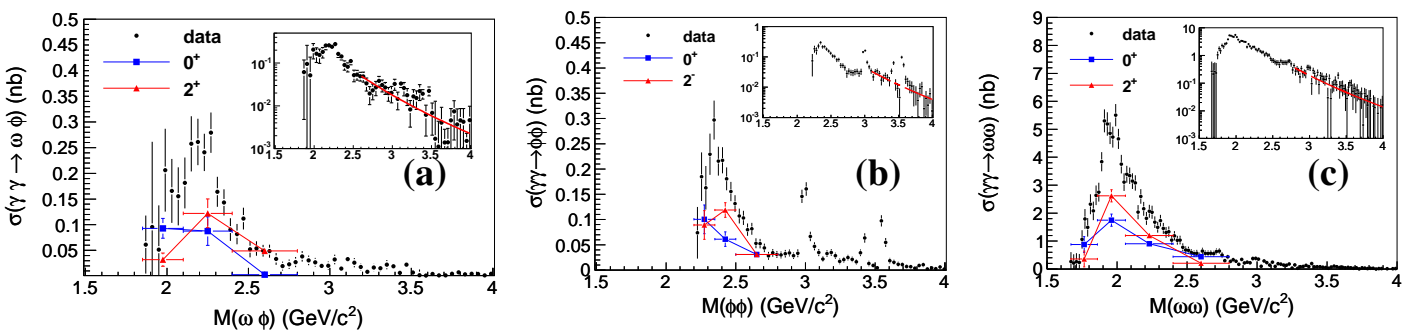

Figure 2: The cross sections of $\gamma \gamma \rightarrow \omega \phi(\mathrm{a}), \phi \phi(\mathrm{b})$, and $\omega \omega$ (c) are shown as points with error bars. The cross sections for different $J^{P}$ values as a function of $M(V V)$ are shown as the triangles and squares with error bars. The inset also shows the cross section on a semi-logarithmic scale. In the high energy region, the solid curve shows a fit to a $W_{\gamma \gamma}^{-n}$ dependence for the cross section after the significant charmonium contributions $\left(\eta_{c}, \chi_{c 0}\right.$ and $\left.\chi_{c 2}\right)$ were excluded.

The cross sections for $\gamma \gamma \rightarrow \omega \phi$ are much lower than the prediction of the $q^{2} \bar{q}^{2}$ tetraquark model [1] of $1 \mathrm{nb}$, while the resonant structure in the $\gamma \gamma \rightarrow \phi \phi$ mode is nearly at the predicted position. However, the $\phi \phi$ cross section is an order of magnitude lower than the expectation in the tetraquark model. On the other hand, the t-channel factorization model [G] predicted that the $\phi \phi$ cross sections vary between $0.001 \mathrm{nb}$ and $0.05 \mathrm{nb}$ in the mass region of $2.0 \mathrm{GeV} / \mathrm{c}^{2}$ to 5.0 $\mathrm{GeV} / c^{2}$, which are much lower than the experimental data. For $\gamma \gamma \rightarrow \omega \omega$, the $\mathrm{t}$-channel factorization model [6] predicted a broad structure between $1.8 \mathrm{GeV} / c^{2}$ and $3.0 \mathrm{GeV} / c^{2}$ with a peak cross section of 10-30 nb near $2.2 \mathrm{GeV} / \mathrm{c}^{2}$, while the one-pion-exchange model [U] predicted an enhancement near threshold around $1.6 \mathrm{GeV} / \mathrm{c}^{2}$ with a peak cross section of $13 \mathrm{nb}$ using a preferred value

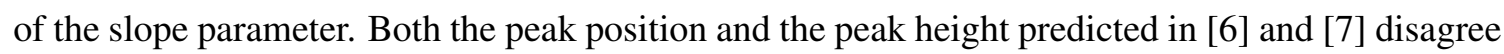
with our measurements.

\section{Charmonium and charmonium-like states}

If $X(3872)$ is a tetraquark state, then it has a $C$-odd parity $(C=-)$ partner, which can dominantly decay into $J / \psi \eta$ and $\chi_{c 1} \gamma . B^{ \pm} \rightarrow(J / \psi \eta(\rightarrow \gamma \gamma)) K^{ \pm}$and $B^{ \pm} \rightarrow\left(\chi_{c 1}(\rightarrow J / \psi \gamma) \gamma\right) K^{ \pm}$decay 
modes are used in the search for $C$-odd partner of the $X(3872)$ and other new narrow resonances. In all the decay modes, $J / \psi$ is reconstructed via $e^{+} e^{-}$and $\mu^{+} \mu^{-} . B$ candidates are identified using energy difference $\Delta E \equiv E_{B}^{*}-E_{\text {beam }}^{*}$ and beam-energy constrained mass $M_{\mathrm{bc}} \equiv \sqrt{\left(E_{\text {beam }}^{*}\right)^{2}-\left(p_{B}^{*}\right)^{2}}$, where $E_{\text {beam }}^{*}$ is the beam energy in the C.M. frame, and $E_{B}^{*}$ and $p_{B}^{*}$ are the energy and momentum of the reconstructed particles in the C.M. frame.

The signal region for $B^{ \pm} \rightarrow J / \psi \eta K^{ \pm}$candidates is defined as $M_{\mathrm{bc}}>5.27 \mathrm{GeV} / c^{2}$ and -35 $\mathrm{MeV}<\Delta E<30 \mathrm{MeV}$. The final $\eta J / \psi$ invariant mass distribution is shown in Fig. [3 together with the fitted results. No hint of a narrow resonance is evident from the current statistics. No $X$ (3872) signal is seen and we obtain the limit $\mathscr{B}\left(B^{ \pm} \rightarrow X(3872) K^{ \pm}\right) \mathscr{B}(X(3872) \rightarrow J / \psi \eta)<3.8 \times 10^{-6}$ at $90 \%$ C.L.

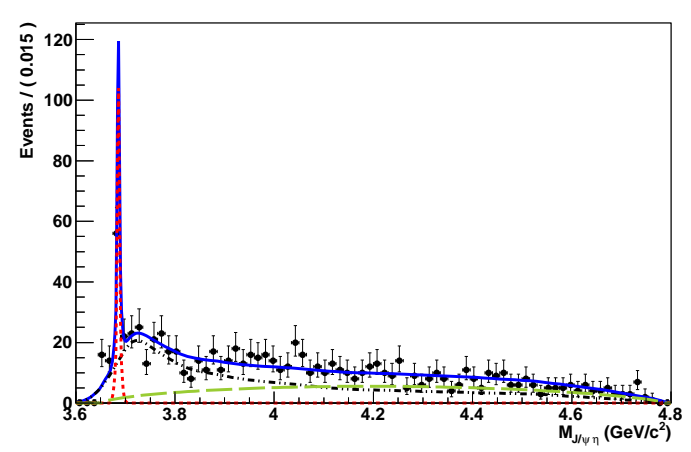

Figure 3: The fit to the $M_{J / \psi \eta}$ distribution. Red dashed (green long dashed) curve shows the signal for $B^{ \pm} \rightarrow \psi^{\prime}(\rightarrow J / \psi \eta) K^{ \pm}$(phase space component $B^{ \pm} \rightarrow J / \psi \eta K^{ \pm}$), while black dashed-dotted curve shows the background parameterized using $B \rightarrow J / \psi X$ MC sample.

Complimentary to $B^{ \pm} \rightarrow(J / \psi \eta) K^{ \pm}$study, the search for the $X$ (3872)'s $C$-odd partner is also carried in $B^{ \pm} \rightarrow\left(\chi_{c 1} \gamma\right) K^{ \pm}$process. Besides the $X(3872)$ 's $C$-odd partner, we also keep an eye on any other possible narrow charmonium or charmonium-like candidate.

After all the event selections, Fig. 团 shows the $M_{\chi_{c 1} \gamma}$ distribution with $M_{\mathrm{bc}}>5.27 \mathrm{GeV} / c^{2}$ (top left), enlarged $M_{\chi_{c 1} \gamma}$ distribution (top right), $M_{\mathrm{bc}}$ distribution with $3.66<M_{\chi_{c 1} \gamma}<3.708 \mathrm{GeV} / c^{2}$ (bottom left), and $M_{\mathrm{bc}}$ distribution with $3.805<M_{\chi_{c 1} \gamma}<3.845 \mathrm{GeV} / c^{2}$ (bottom right). The dots with error bars are data, and the blue solid lines are the projections from $2 \mathrm{D}$ unbinned maximum likelihood fits. No $X(3872)$ signal is seen and the limit $\mathscr{B}\left(B^{ \pm} \rightarrow X(3872) K^{ \pm}\right) \mathscr{B}\left(X(3872) \rightarrow \gamma \chi_{c 1}\right)<$ $2.0 \times 10^{-6}$ at $90 \%$ C.L. is obtained. Besides the clear $\psi^{\prime}$ signal, we find a clear evidence of a nar-

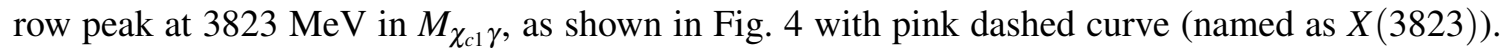
The signal significance is $4.2 \sigma$ with systematic error included. The mass and width of this peak are estimated to be $3823.5 \pm 2.1 \mathrm{MeV} / c^{2}$ and $4 \pm 6 \mathrm{MeV}$, respectively. We noticed that charmonium model predicts a narrow state $\left({ }^{3} D_{2} c \bar{c}\right)$ at around $3810-3840 \mathrm{MeV} / c^{2}$ [[]]. So the $X(3823)$ is probably $\psi_{2}$ state. The measured $\mathscr{B}\left(B^{ \pm} \rightarrow X(3823) K^{ \pm}\right) \mathscr{B}\left(X(3823) \rightarrow \gamma \chi_{c 1}\right)$ is $\left(9.70_{-2.52-1.03}^{+2.84+1.06}\right) \times 10^{-6}$, where the first errors are statistical and the second systematic.

\section{Bottomonium and bottomonium-like states}

The spin-singlet states $h_{b}(n P)$ and $\eta_{b}(n S)$ alone provide information concerning the spin-spin 

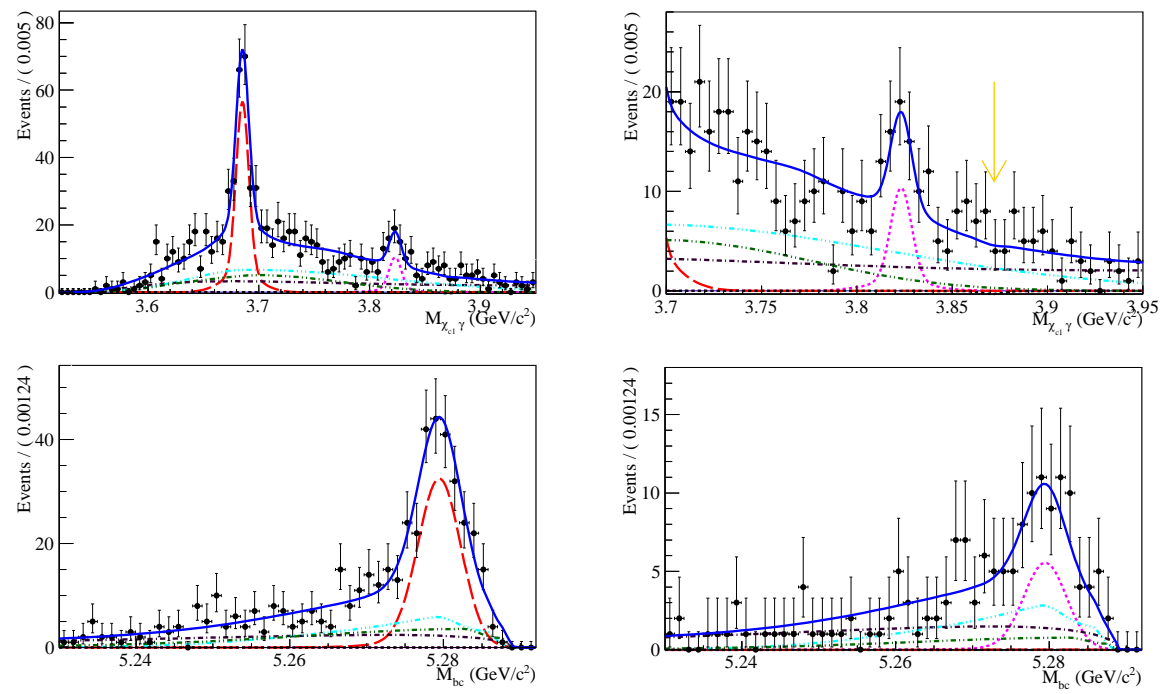

Figure 4: The projections from 2D unbinned maximum likelihood fits to the $M_{\chi_{c 1} \gamma}$ distribution with $M_{\mathrm{bc}}>$ $5.27 \mathrm{GeV} / c^{2}$ (top left), enlarged $M_{\chi_{c 1} \gamma}$ distribution (top right, yellow arrow shows the $X(3872)$ position), $M_{\mathrm{bc}}$ distribution with $3.66<M_{\chi_{c 1} \gamma}<3.708 \mathrm{GeV} / c^{2}$ (bottom left), and $M_{\mathrm{bc}}$ distribution with $3.805<M_{\chi_{c 1} \gamma}<$ $3.845 \mathrm{GeV} / c^{2}$ (bottom right). The dots with error bars are data. The blue solid line is form the overall fit. The red large-dashed and pink dashed curves are for $\psi^{\prime}$ and $\psi_{2}$ signals, respectively, while black dotted-dashed, dark green two dotted-dashed, and cyan three dotted-dashed curves are for the combinatorial background, $B^{ \pm} \rightarrow \psi^{\prime}$ (other than $\left.\chi_{c 1} \gamma\right) K^{ \pm}$events, and peaking background component, respectively.

(or hyperfine) interaction in bottomonium. Measurements of the $h_{b}(n P)$ masses provide unique access to the $P$-wave hyperfine splitting, $\Delta M_{\mathrm{HF}} \equiv\left\langle M\left(n^{3} P_{J}\right)\right\rangle-M\left(n^{1} P_{1}\right)$, the difference between the spin-weighted average mass of the $P$-wave triplet states $\left(\chi_{b J}(n P)\right.$ or $\left.n^{3} P_{J}\right)$ and that of the corresponding $h_{b}(n P)$, or $n^{1} P_{1}$. We use a $121.4 \mathrm{fb}^{-1}$ data sample collected near the peak of the $\Upsilon(5 S)$ resonance $(\sqrt{s} \sim 10.865 \mathrm{GeV})$ with the Belle detector to report the first observation of the $h_{b}(1 P)$ and $h_{b}(2 P)$ produced via $e^{+} e^{-} \rightarrow h_{b}(n P) \pi^{+} \pi^{-}$in the $\Upsilon(5 S)$ region [ [ $]$ ].

We observe the $h_{b}(n P)$ states in the $\pi^{+} \pi^{-}$missing mass spectrum of hadronic events. The $\pi^{+} \pi^{-}$missing mass is defined as $M_{\text {miss }}^{2} \equiv\left(P_{\Upsilon(5 S)}-P_{\pi^{+} \pi^{-}}\right)^{2}$, where $P_{\Upsilon(5 S)}$ is the 4-momentum of the $\Upsilon(5 S)$ determined from the beam momenta and $P_{\pi^{+} \pi^{-}}$is the 4-momentum of the $\pi^{+} \pi^{-}$system. The $\pi^{+} \pi^{-}$transitions between $\Upsilon(n S)$ states provide high-statistics reference signals.

To reconstruct the $\Upsilon(5 S) \rightarrow h_{b}(n P) \pi^{+} \pi^{-}$transitions inclusively, we use a general hadronic event selection. The $M_{\text {miss }}$ spectrum after subtraction of both the combinatoric and $K_{S}^{0} \rightarrow \pi^{+} \pi^{-}$ contributions is shown with the fitted signal functions overlaid in Fig. [5]. The significances of the $h_{b}(1 P)$ and $h_{b}(2 P)$ signals, with systematic uncertainties accounted for, are $5.5 \sigma$ and $11.2 \sigma$, respectively. The measured masses of $h_{b}(1 P)$ and $h_{b}(2 P)$ are $M=\left(9898.2_{-1.0-1.1}^{+1.1+1.0}\right) \mathrm{MeV} / c^{2}$ and $M=$ $\left(10259.8 \pm 0.6_{-1.0}^{+1.4}\right) \mathrm{MeV} / c^{2}$, respectively. Using the world average masses of the $\chi_{b J}(n P)$ states, we determine the hyperfine splittings to be $\Delta M_{\mathrm{HF}}=(+1.7 \pm 1.5) \mathrm{MeV} / c^{2}$ and $\left(+0.5_{-1.2}^{+1.6}\right) \mathrm{MeV} / c^{2}$, respectively, where statistical and systematic uncertainties are combined in quadrature.

The observation of anomalously high rates for $\Upsilon(5 S) \rightarrow \Upsilon(n S) \pi^{+} \pi^{-}(n=1,2,3)$ and $\Upsilon(5 S) \rightarrow$ $h_{b}(n P) \pi^{+} \pi^{-}(m=1,2)$ transitions suggests that exotic mechanisms are contributing to $\Upsilon(5 S)$ de- 


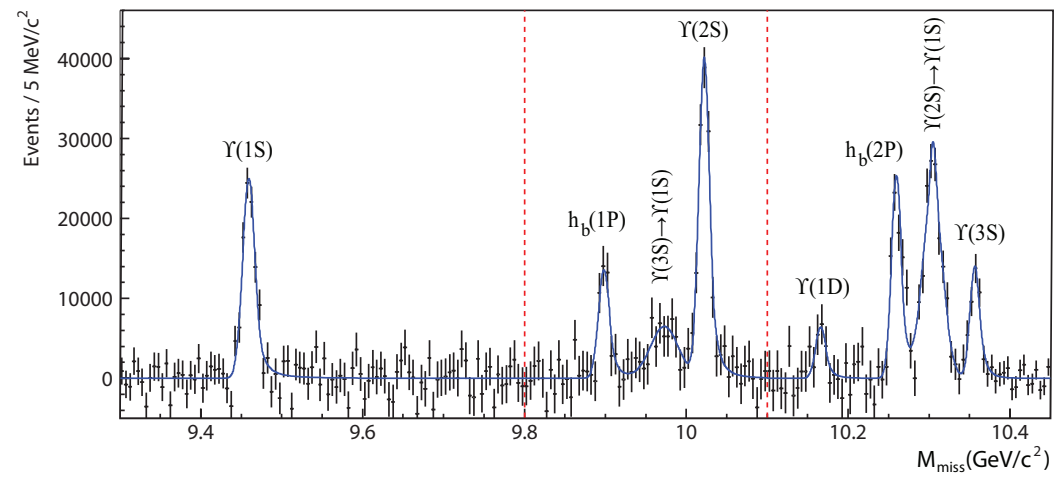

Figure 5: The inclusive $M_{\text {miss }}$ spectrum with the combinatoric background and $K_{S}^{0}$ contribution subtracted (points with errors) and signal component of the fit function overlaid (smooth curve). The vertical lines indicate boundaries of the fit regions.

cays. Amplitude analyses of the three-body $\Upsilon(5 S) \rightarrow \Upsilon(n S) \pi^{+} \pi^{-}$decays with $\Upsilon(n S) \rightarrow \mu^{+} \mu^{-}$ are performed by means of unbinned maximum likelihood fits to two-dimensional $M^{2}\left[\Upsilon(n S) \pi^{+}\right]$ vs. $M^{2}\left[\Upsilon(n S) \pi^{-}\right]$Dalitz distributions [ए]]. One-dimensional invariant mass projections for events in the $\Upsilon(n S)$ signal regions are shown in Fig. 6 , where two peaks are observed in the $\Upsilon(n S) \pi$ system near $10.61 \mathrm{GeV} / c^{2}$ and $10.65 \mathrm{GeV} / c^{2}$ (named as $Z_{b}(10610)$ and $Z_{b}(10650)$ ). The combined statistical significance of the two peaks exceeds $10 \sigma$ for all $\Upsilon(n S) \pi^{+} \pi^{-}$channels.
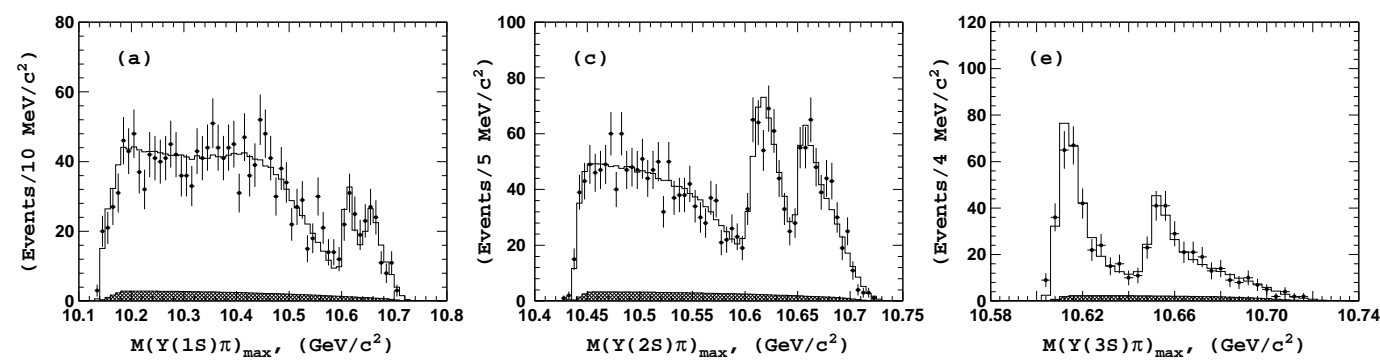

Figure 6: Comparison of fit results (open histogram) with experimental data (points with error bars) for events in the (a) $\Upsilon(1 S)$, (c) $\Upsilon(2 S)$, and (e) $\Upsilon(3 S)$ signal regions. The hatched histogram shows the background component.

To study the resonant substructure of the $\Upsilon(5 S) \rightarrow h_{b}(n P) \pi^{+} \pi^{-}(m=1,2)$ decays we measure their yield as a function of the $h_{b}(1 P) \pi^{ \pm}$invariant mass. The decays are reconstructed inclusively using the missing mass of the $\pi^{+} \pi^{-}$pair, $M_{\text {miss }}$. We fit the $M_{\text {miss }}$ spectra in bins of $h_{b}(1 P) \pi^{ \pm}$ invariant mass, defined as the missing mass of the opposite sign pion, $M_{\text {miss }}\left(\pi^{\mp}\right)$. We combine the $M_{\text {miss }}$ spectra for the corresponding $M_{\text {miss }}\left(\pi^{+}\right)$and $M_{\text {miss }}\left(\pi^{-}\right)$bins and we use half of the available $M_{\text {miss }}(\pi)$ range to avoid double counting.

The results for the yield of $\Upsilon(5 S) \rightarrow h_{b}(n P) \pi^{+} \pi^{-}(m=1,2)$ decays as a function of the $M_{\text {miss }}(\pi)$ are shown in Fig. $\mathbb{Z}$, where the fit results are shown as solid histograms. The two-peak structures are clear in both of them. The default fit hypothesis is favored over the phase-space fit hypothesis at the $18 \sigma[6.7 \sigma]$ level for the $h_{b}(1 P)\left[h_{b}(2 P)\right]$. 
Weighted averages over all five channels give $M=10607.2 \pm 2.0 \mathrm{MeV} / c^{2}, \Gamma=18.4 \pm 2.4 \mathrm{MeV}$ for the $Z_{b}(10610)$ and $M=10652.2 \pm 1.5 \mathrm{MeV} / c^{2}, \Gamma=11.5 \pm 2.2 \mathrm{MeV}$ for the $Z_{b}(10650)$, where statistical and systematic errors are added in quadrature. Angular analysis favors a $J^{P}=1^{+}$assignment for both $Z_{b}^{+}$states, which must also have negative $G$-parity. Transitions through $Z_{b}^{+}$to the $h_{b}(n P)$ saturate the observed $\pi^{+} \pi^{-} h_{b}(n P)$ cross sections. The two masses of $Z_{b}^{+}$states are just a few $\mathrm{MeV}$ above the $B^{*} \bar{B}$ and $B^{*} \bar{B}^{*}$ thresholds, respectively. The $Z_{b}^{+}$cannot be simple mesons because they are charged and have $b \bar{b}$ content.
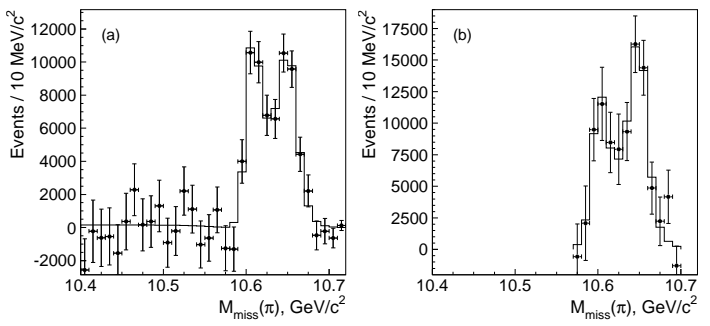

Figure 7: The (a) $h_{b}(1 P)$ and (b) $h_{b}(2 P)$ yields as a function of $M_{\text {miss }}(\pi)$ (points with error bars) and results of the fit (histogram).

\section{Summary}

We reviewed here some recent results on exotic states at the Belle experiment, including the measurement of the cross sections of $\gamma \gamma \rightarrow \omega \phi, \phi \phi$, and $\omega \omega$; the search for the $X(3872)$ 's C-odd partner in $\eta J / \psi$ and $\gamma \chi_{c 1}$ modes in $B$ decays; the evidence of $\psi_{2}$ in $B^{ \pm} \rightarrow K^{ \pm} \gamma \chi_{c 1}$; the observation of $h_{b}(1 P), h_{b}(2 P), \Upsilon(1 D)$, and two charged $Z_{b}(10610)$ and $Z_{b}(10650)$ states.

\section{References}

[1] S. Uehara et al. (Belle Collaboration), Phys. Rev. Lett. 104, 092001 (2010).

[2] C. P. Shen et al. (Belle Collaboration), Phys. Rev. Lett. 104, 112004 (2010).

[3] Z. Q. Liu et al. (Belle Collaboration), Phys. Rev. Lett. 108, 232001 (2012).

[4] Using $\omega \phi$ as an example, $z$ is the cosine of the scattering polar angle of $\phi$ in the $\gamma \gamma$ C.M. system; $z^{*}$ and $\phi^{*}$ are the cosine of the helicity angle of $K^{+}$in the $\phi$ decays and the azimuthal angle defined in the $\phi$ rest frame with respect to the $\gamma \gamma \rightarrow \omega \phi$ scattering plane; $z^{* *}$ and $\phi^{* *}$ are the cosine of the helicity angle of normal direction to the decay plane of the $\omega \rightarrow \pi^{+} \pi^{-} \pi^{0}$ and the azimuthal angle defined in the $\omega$ rest frame.

[5] N. N. Achasov and G. N. Shestakov, Usp. Fiz. Nauk 161, 53 (1991) [Sov. Phys. Usp. 34, 471 (1991)].

[6] G. Alexander, A. Levy and U. Maor, Z. Phys. C 30, 65 (1986).

[7] N. N. Achasov, V. A. Karnakov and G. N. Shestakov, Z. Phys. C 36, 661 (1987).

[8] S. Godfrey and N. Isgur, Phys. Rev. D 32, 189 (1985); E. Eichten et al., Phys. Rev. D 89, 162002 (2002); E. Eichten et al., Phys. Rev. D 69, 094019 (2004).

[9] I. Adachi et al. (Belle Collaboration), Phys. Rev. Lett. 108, 032001 (2012).

[10] A. Bondar et al. (Belle Collaboration), Phys. Rev. Lett. 108, 122001 (2012). 